\title{
Validation of NIR Model for On-line Monitoring of Flos Lonicera Japonica Extraction Process with Different Batches of Materials
}

\author{
http://dx.doi.org/10.3991/ijoe.v9i4.2934 \\ Chenglin Sui ${ }^{1,2}$, Zhisheng Wu ${ }^{1,2}$, Yanfang Peng ${ }^{1,2}$, Luwei Zou ${ }^{1,2}$, Yanling Pei ${ }^{1,2}$, \\ Xinyuan Shi ${ }^{1,2^{*}}$, Yanjiang Qiao ${ }^{1,2^{*}}$ \\ ${ }^{1}$ Beijing University of Chinese Medicine, Beijing, China \\ ${ }^{2}$ The Key Laboratory of TCM-Information Engineering of State Administration of Traditional Chinese Medicine, \\ Beijing, China
}

\begin{abstract}
In this study, on-line NIRs (Near Infrared Spectroscopy) monitoring technology, as a useful tool, has been verified during the extraction process of Flos Lonicera Japonica. To gain representative samples and accurate model result, the detrimental effects from NIR collecting circumstance were significantly reduced with meticulous designs. Based on what, different batches of Flos Lonicera Japonica material were real-time monitored in the extraction process. 10 design schemes were used to verify whether or not different batches could disturb predictive results of the quantitative model. According to a series of chemometric indicators, such as SEC, SECV, SEP, RPD, it is concluded that PLS models established by samples of different batches of raw herbs from the same source could be used to monitor the extraction process with acceptable accuracy. Our research has demonstrated that on-line NIRs monitoring technology as a fast, non-destructive and realtime monitoring tool could be utilized in traditional Chinese medicine preparation.
\end{abstract}

Index Terms-Batch-to-batch reproducibility, Extraction process, Near infrared Spectroscopy, On-line monitoring1. Introduction

\section{INTRODUCTION}

In order to enhance the understanding of the manufacturing process, U.S. Food and Drug Administration published a regulatory framework (Process Analytical Technology, PAT) in 2004. The online process monitoring is a system for the design, analysis, and control of pharmaceutical manufacturing processes, based on timely measurements of critical qualities and performance attributes of in-process materials and products [1].

Near Infrared Spectroscopy (NIRs) has received considerable interest within the pharmaceutical industry and is generally chosen as a PAT tool [2]. It offers the possibility for the pharmaceutical analyst to perform component analysis in a real time basis and in a fast and non-destructive manner, requiring little or no further sample preparation.

The implementation of online quality control in the medicine production is the trend of TCM manufacturing $[3,4,5]$. As one of the most popular techniques, extraction is widely used in the preparation process of traditional Chinese medicine (TCM). In our study, to ensure representative samples, the detrimental effects from NIR collecting circumstance were significantly reduced with meticulous design such as on-line NIR spectrum measurement system and sampling device. Based on on-line equipment, predictive capability of NIR quantitative models established by samples from different batches of material would be fully discussed here.

In this paper, the extraction process of different batches of Flos Lonicera Japonica was analyzed by an on-line NIR system. Novel experimental schemes among batch-tobatch were devised to compare predictive results of partial least squares (PLS) models. We were interested in whether there would be a large discrepancy among analytical models established by different batches. The purpose of this paper provided an approximate reference approach about the on-line NIR model among batches.

\section{MATERIALS AND METHODS}

\section{A. Materials}

Two batches of Flos Lonicera Japonica (Batch A: Lot No. 120308, Batch B: Lot No. 120330) were provided by Yabao Beizhongda (Beijing) Pharmaceutical Co., Ltd. Chlorogenic acid reference standard (Lot No. 110753200413) was supplied by the National Institute for the Control of Pharmaceutical and Biological Products (Beijing, China). Methanol (Fisher Scientific, USA) and acetonitrile (Fisher Scientific, USA) were of HPLC grade. Ethanol was analytical grade (Beijing Chemical Works, Beijing, China). Phosphoric acid was of analytical grade (Beijing Chemical Works, Beijing, China). Deionized water was purified by Milli-Q water system (Millipore Corp., Bedford, MA, USA).

\section{B. Process Description and Sampling Circumstance}

Flos Lonicera Japonica was extracted with pure water in a $100 \mathrm{~L}$ multi-functional extractor. $8 \mathrm{Kg}$ Flos Lonicera Japonica was firstly immersed in water for $30 \mathrm{~min}$, and then heated and kept under boiling for $30 \mathrm{~min}$. The stirring paddle was set at the speed of $50 \mathrm{rpm}$. After extracts boiled, the NIR spectra were collected every $5 \mathrm{~min}$, while in other periods, NIR spectra were obtained every $3 \mathrm{~min}$. Once the scanning was completed, the tap was open and the sample was collected for following analysis by HPLC. This extraction process was conducted with Batch A (Experiment a) and Batch B (Experiment b, c, d). 


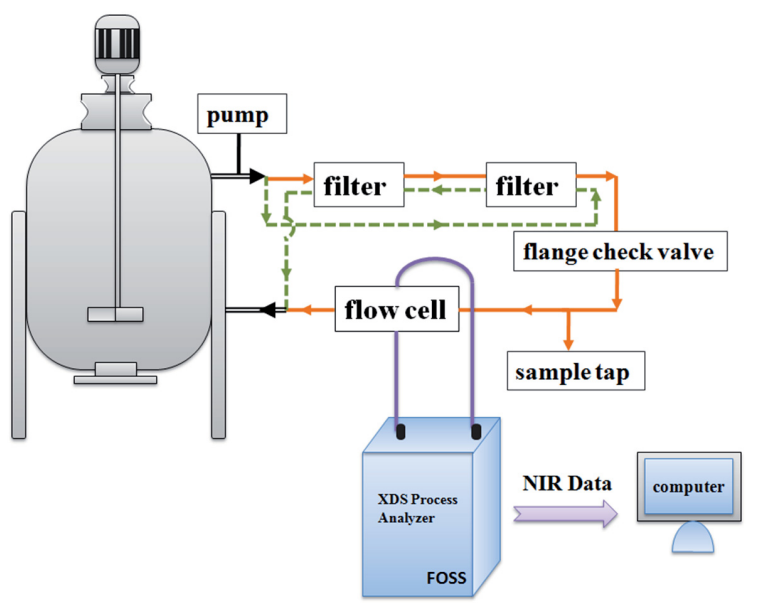

Figure 1. The scheme of an external cycle system. Arrows point to the direction of extracts; the dashed lines represent the backwashing system; solid lines represent the pipeline rapid loop; double lines represent public pipes; and the optical probe connect flow cell with process analyzer.

On-line NIR models can be affected, to some extent, by air bubble, solid impurities, temperature, etc [6]. To overcome the influences, all samples were obtained by external cycle system external, which included on-line NIR scanning system and the sampling device (Figure 1). The sampling device consists of 5 parts: motive force supply system, pipeline rapid loop, two filters $(150 \mu \mathrm{m}$, $180 \mu \mathrm{m})$, backwashing system and flange check valve. Specific functionalities of each part were shown as following:

The motive force supply system mainly relied on the pneumatic pump which propelled liquid extract in the external cycle. To avoid on-line NIR spectra influenced by solid impurities, the extracts passed through a $100 \mu \mathrm{m}$ strainer before entering the external cycle system. Two extra external filters $(150 \mu \mathrm{m}, 180 \mu \mathrm{m})$ were used to protect against solid content. Besides, the external filters can work as two buffer containers to reduce bubbles and turbulence. In order to prevent the filters from being blocked by solid impurities, backwashing system was used to flush them out by compressed air and reverse flow of extracts. Finally, flange check valve effectively remove turbulent fluid.

\section{NIR Equipment and Software}

The on-line NIR spectra were collected by two fiber optic probes. NIR radiation through $2 \mathrm{~mm}$ optical path was the transmission mode with the XDS process analyzer and VISION software (Foss NIRSystems, Silver Spring, MD, USA). The range of spectra was between $800 \mathrm{~nm}$ and $2200 \mathrm{~nm}$. Spectra were an average of 32 scans with a wavelength increment of $0.5 \mathrm{~nm}$. Data analysis was performed by VISION software (Vision 3.5, Foss, Silver Spring, MD, USA).

\section{Reference Method}

All the samples were diluted with 50\% methanol-water solution and the Chlorogenic acid content was accurately determined by using HPLC which was recommended by the Chinese Pharmacopoeia (ChP, 2010 Edition) for Flos Lonicera Japonica. A SHIMADZU HPLC apparatus, equipped with a LC-20AT system, an auto sampler and a DAD detector was used. The concentration of chlorogenic acid was analyzed by reverse-phase chromatography on an Sunfire ${ }^{\circledR} \mathrm{C}_{18}$ column $(250 \mathrm{~mm} \times 4.6 \mathrm{~mm}, 5 \mu \mathrm{m}$, Waters $)$ with isocratic elution of the mobile phase consisted of acetonitrile and water with $0.4 \%$ Phosphoric acid (13: 87 , $\mathrm{v} / \mathrm{v}$ ) with a flow rate of $1.0 \mathrm{~mL} / \mathrm{min}$ at $30{ }^{\circ} \mathrm{C}$, and detection wavelength at $327 \mathrm{~nm}$ were set.

\section{E. Model building}

For comprehending predictive model performance of extracts from different batches of raw material, ten different combinations about calibration and validation among experiments were designed. In the 10 schemes, whole combinations about predicting between different batches raw material were involved. Table 1 has presented the schemes in detail.

TABLE I.

SAMPLES IN CALIBRATION AND VALIDATION SETS

\begin{tabular}{ccccc}
\hline \multirow{2}{*}{ Scheme } & \multicolumn{2}{c}{ Calibration } & \multicolumn{2}{c}{ Validation } \\
\cline { 2 - 5 } & Samples & Experiments ID & Samples & Experiments ID \\
\hline I & 59 & b, d & 27 & $\mathrm{c}$ \\
II & 57 & b, c & 29 & $\mathrm{~d}$ \\
III & 56 & $\mathrm{c}, \mathrm{d}$ & 30 & $\mathrm{~b}$ \\
IV & 59 & $\mathrm{~b}, \mathrm{~d}$ & 29 & $\mathrm{a}$ \\
V & 57 & $\mathrm{~b}, \mathrm{c}$ & 29 & $\mathrm{a}$ \\
VI & 56 & $\mathrm{c}, \mathrm{d}$ & 29 & $\mathrm{a}$ \\
VII & 86 & $\mathrm{~b}, \mathrm{c}, \mathrm{d}$ & 29 & $\mathrm{a}$ \\
VIII & 88 & $\mathrm{a}, \mathrm{b}, \mathrm{d}$ & 27 & $\mathrm{c}$ \\
IX & 86 & $\mathrm{a}, \mathrm{b}, \mathrm{c}$ & 29 & $\mathrm{~d}$ \\
$\mathrm{X}$ & 85 & $\mathrm{a}, \mathrm{c}, \mathrm{d}$ & 30 & $\mathrm{~b}$ \\
\hline
\end{tabular}

\section{RESULT AND DisCUSSION}

\section{A. HPLC Determination of Chlorogenic Acid}

According to the Chinese Pharmacopoeia(2010 Edition, vol. I), the reference values obtained by HPLC were accurate and could be used in NIR calibration model. Figure 2 showed typical HPLC chromatograms of chlorogenic acid in reference solution and sample solution. The calibration curve showed good linearity $(\mathrm{R}=$ $0.9999)$ within the ranging from $0.0792 \mu \mathrm{g}$ to $0.792 \mu \mathrm{g}$. Distribution of chlorogenic acid concentration had a similar trend in the extraction process, which was presented in Figure 3.
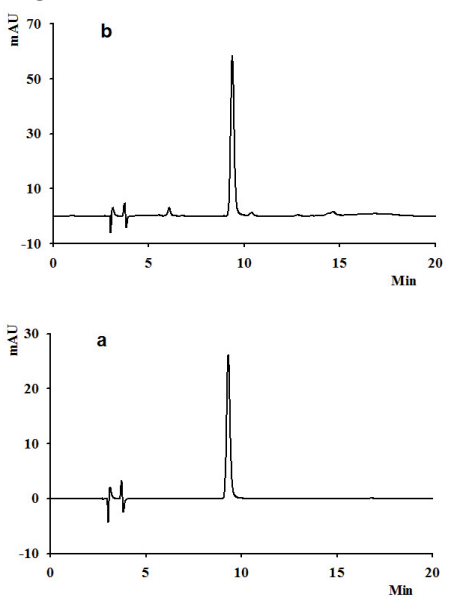

Figure 2. The chromatograms of (a) chlorogenic acid reference standard and (b) a sample solution in extraction process. 


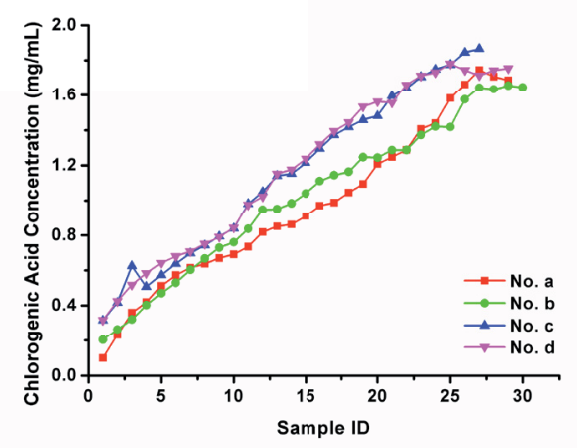

Figure 3. Figure 3. Tendency of chlorogenic acid concentration in 4 experiments

\section{B. Variable Selection and NIR Spectra Pretreatment}

Since the NIR spectra were collected by using an online sampling system, interferences were reduced but could not be completely avoided. From the score plots of the first two principal components (see in Figure 4), most of the samples formed one cluster, and only two samples were far away from the cluster and thus called outliers. To establish a robust calibration model, the outliers need to be removed.

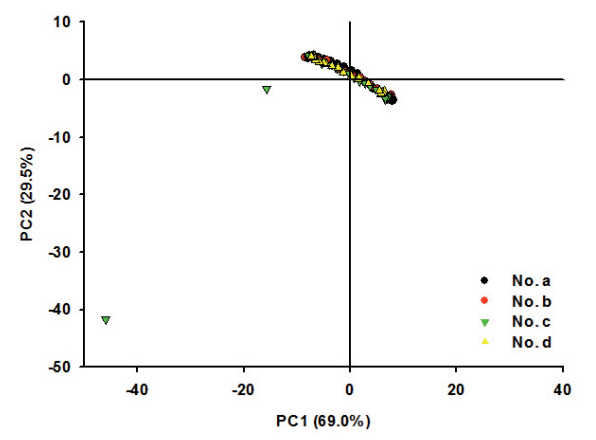

Figure 4. Figure 4. The PC1-PC2 scattergram of all the samples from 4 experiments.

The original NIR spectra of the sample solutions were shown in Figure 5. There was a large fluctuation in the combination region $(2000 \mathrm{~nm} \sim 2200 \mathrm{~nm})$ [7]. In addition, the position of the intense absorbance of the aqueous solution is located near $1940 \mathrm{~nm}[8,9]$. Thus, to improve the accuracy of the calibration models, the spectral region of 800-1900 nm was selected for the following analysis.

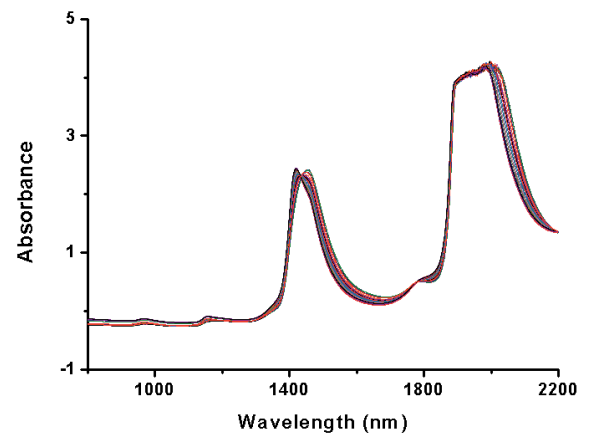

Figure 5. Figure 5. Raw NIR spectra of one of the researched experiments (No. a)
On the purpose of establishing a stable PLS calibration model, cross validation was executed with a segment size of four, and a PRESS plot (Figure 6) was produced. The optimum number of latent variables was determined by the first minimum PRESS value with the best prediction for the cross validation in the PRESS plot. Obviously, raw spectra was superior to others for PLS model. Consequently, the original spectra were utilized to build PLS model in this study.

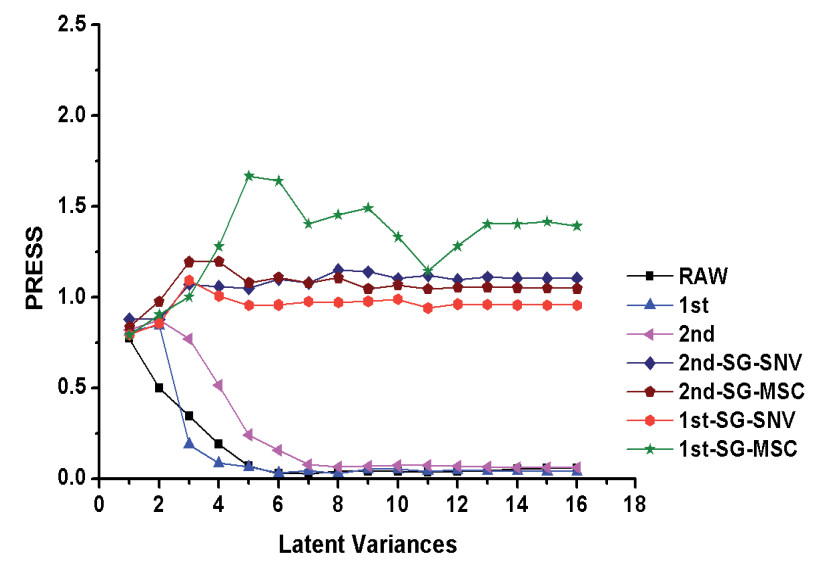

Figure 6. Figure 6. PRESS plot in the NIR spectra range of 800-1900nm

\section{Predictive Result of PLS Models}

To select the best equations, some statistics were assessed in terms of $R^{2}, \mathrm{SEC}, \mathrm{SECV}, \mathrm{SEP}[10], \mathrm{RPD}$, and CV $[11,12]$. A good model should have a lower SEC, a lower SECV and a higher determination coefficient, but also a small difference between SEC and SECV. The other statistics, such as RPD, CV, or ratio between SEC and SEP, enable standardization of the SEP, facilitating the comparison of results obtained with sets of different means. All of parameters in calibration and validation were shown in Table 2.

Among the first six schemes mentioned above, although the predictive statistics (SEP and RPD) in scheme $\mathrm{V}$ and scheme VI were a bit lower than the same statistics in the first three schemes (I, II, and III), there was a better predictive performance in scheme IV $\left(R^{2}=\right.$ $0.9938, \mathrm{SEP}=0.0358, \mathrm{RPD}=13.1)$ than in schemes I, II, and III. It was concluded that the stability of models was not disturbed by different experiments. Moreover, the results of all the six schemes were favorable, indicating the efficiency and robustness of PLS models built by different batches of material.

In scheme VII, the calibration set composed of Batch B was validated by Batch A. From a series of chemometric indicators $(\mathrm{SEP}=0.0474, \mathrm{SEC} / \mathrm{SEP}=0.84, \mathrm{RPD}=9.8)$ in scheme VII, the PLS model fitting result indicated that the calibration model had satisfactory robustness and predictive ability. Furthermore, the predictive result of different batches was as good as those of the same batch of material. In schemes VII to $X$, samples in the calibration sets were enlarged by adding samples from one experiment, and the result showed no obvious change. 
PAPER

VALIDATION OF NIR MOdEL FOR ON-LINE MONITORING OF FlOS LONICERA JAPONICA EXTRACTION PROCESS WITH...

TABLE II.

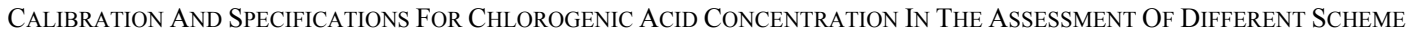

\begin{tabular}{cccccccccc}
\hline scheme & Factors & $\boldsymbol{R}_{\text {cal }}$ & SEC & SECV & $\boldsymbol{R}_{\text {val }}^{2}$ & SEP & SEC/SEP & RPD & CV (\%) \\
\hline I & 7 & 0.9955 & 0.0332 & 0.0360 & 0.9878 & 0.0519 & 0.64 & 9.2 & 4.8 \\
II & 7 & 0.9945 & 0.0365 & 0.0424 & 0.9928 & 0.0397 & 0.92 & 12.0 & 3.2 \\
III & 6 & 0.9944 & 0.0378 & 0.0429 & 0.9888 & 0.0461 & 0.82 & 9.7 & 4.6 \\
IV & 7 & 0.9955 & 0.0332 & 0.0360 & 0.9938 & 0.0358 & 0.93 & 13.0 & 3.6 \\
V & 7 & 0.9945 & 0.0365 & 0.0424 & 0.9851 & 0.0558 & 0.65 & 8.3 & 5.8 \\
VI & 6 & 0.9944 & 0.0378 & 0.0429 & 0.9754 & 0.0715 & 0.53 & 6.5 & 8.1 \\
VII & 7 & 0.9933 & 0.0399 & 0.0451 & 0.9892 & 0.0474 & 0.84 & 9.8 & 4.9 \\
VIII & 8 & 0.9950 & 0.0345 & 0.0389 & 0.9845 & 0.0585 & 0.59 & 8.2 & 5.3 \\
IX & 7 & 0.9919 & 0.0432 & 0.0469 & 0.9942 & 0.0358 & 1.21 & 13.3 & 3.0 \\
X & 7 & 0.9926 & 0.0431 & 0.0495 & 0.9884 & 0.0471 & 0.92 & 9.4 & 4.5 \\
\hline
\end{tabular}

The parameters in the models have revealed that the extraction process can be monitored by an on-line NIR collecting approach and the PLS qualitative models were reliable. Generally speaking, to ensure the reproducibility between different batches of TCM final products, most pharmaceutical manufacturers purchase crude materials from the same source. Hence, there wasn't significant difference emerged among different batches of raw herb. To acquire representative samples, a stable environment was provided by the on-line NIR analyzer and the sampling system in our research, which contributed greatly to model accuracy. It was the reason why raw materials from different batches were not able to impact PLS models.

\section{CONCLUSION}

This research has successfully settled the puzzle about the reliability of the on-line NIR spectrum technology for monitoring the extraction process of TCM. In the present study, stable NIR collecting circumstance (the on-line NIR instrument and entire sampling device) improved the predictive capability of models. A valuable method on building calibration models has been established to monitor the extraction process.

In our research, by using an elaborately designed spectrum scanning system, air bubbles, flow rate, and other influences were reduced, and the quality of NIR spectra was improved. All of these projects were applied in the extraction process, and NIRs were successfully used as a fast, non-destructive and real-time monitoring tool in the TCM research. of models. A valuable method on building calibration models has been established to monitor the extraction process.

\section{ACKNOWLEDGEMENTS}

The authors wish to acknowledge research funding supports from "the National Major Projects of Science and Technology named 'Creation of Major New Drugs'" (No. 2010ZX09502-002, China) and Scientific Research Project of Beijing University of Chinese Medicine (No. JYB22-XS034).

\section{REFERENCES}

[1] http: //www.fda.gov/ downloads/ Drugs/ Guidance Compliance Regulatory Information/Guidances /UCM070305. pdf.

[2] Y. Roggo, P. Chalus, L. Maurer, C. Lema-Martinez, A. Edmond, and N. Jent, " A review of near infrared spectroscopy and chemometrics in pharmaceutical technologies," J. Pharm. Biomed. Anal., vol.44, pp. 683-700, 2007. http://dx.doi.org/10.1016/j.jpba.2007.03.023

[3] Y.J. Wu, Y. Jin, Y.R. Li, D. Sun, X.S. Liu, and Y. Chen, "NIR spectroscopy as a process analytical technology (PAT) tool for on-line and real-time monitoring of an extraction process," $V i b$. Spectrosc., vol.58, $\quad$ pp. 109-118, 2012. http://dx.doi.org/10.1016/j.vibspec.2011.10.006

[4] Y.J. Wu, Y. Jin, H.Y. Ding, L.J. Luan, Y. Chen, and X.S. Liu, "In-line monitoring of extraction process of scutellarein from Erigeron breviscapus (vant.) Hand-Mazz based on qualitative and quantitative uses of near-infrared spectroscopy," Spectrochim. Acta A., vol.79, pp. 934-939, 2011. http://dx.doi.org/10.1016/j.saa.2011.03.056

[5] B. Xu, Z.S. Wu, Z.Z. Lin, C.L. Sui, X.Y. Shi, and Y.J. Qiao, "NIR analysis for batch process of ethanol precipitation coupled with a new calibration model updating strategy," Anal. Chim. Acta, vol.720, $\quad$ pp. $22-28, \quad 2012$. http://dx.doi.org/10.1016/j.aca.2012.01.022

[6] Z.S. Wu, M. Du, B. Xu, Z.Z. Lin, X.Y. Shi, and Y.J. Qiao, "Absorption characteristics and quantitative contribution of overtones and combination of NIR: Method development and validation," J. Mol. Struct., vol.1019, pp. 97-102, 2012. http://dx.doi.org/10.1016/j.molstruc.2012.03.058

[7] H. Yamatera, B.Fitzpatrick, and G. Gordok, "Near infrared spectra of water and aqueous solutions," J. Mol. Struct., pp. 268278, 1964.

[8] G.R. Choppin, and J.R. Downey, "Near-Infrared studies of the structure of water. IV. water in relatively nonpolar solvents," Choppin, J. Chem. Phys., vol.56, pp. 5890, 1972. http://dx.doi.org/10.1063/1.1677133

[9] P. Paz, M.-T. Sánchez, D. Pérez-Marín, J.-E. Guerrero, and A. Garrido-Varo, "Evaluating NIR instruments for quantitative and qualitative assessment of intact apple quality,"J. Sci. Food Agric., vol.89, pp. 781-790, 2009. http://dx.doi.org/10.1002/jsfa.3512

[10] V. Bellon-Maurel, E. Fernandez-Ahumada, B. Palagos, J.-M. Roger, and A. McBratney, "Critical review of chemometric indicators commonly used for assessing the quality of the prediction of soil attributes by NIR spectroscopy," Trends Analyt. Chem., vol.29, pp. $1073-1081,2010$. http://dx.doi.org/10.1016/j.trac.2010.05.006

[11] Z. Wu, B. Xu, M. Du, C. Sui, X. Shi, and Y. Qiao, "Validation of a NIR quantification method for the determination of chlorogenic acid in Lonicera japonica solution in ethanol precipitation process," J. Pharm. Biomed. Anal., vol.62, pp. 1-6, 2012. http://dx.doi.org/10.1016/j.jpba.2011.12.005

[12] P.J.d.G. H. Swierenga, A.P. de Weijer, and M.W.J. Derksen, L.M.C. Buydens, "Improvement of PLS model transferability by robust wavelength selection," Chemom. Intell. Lab. Syst., vol.41, pp. 237-248, 1998. http://dx.doi.org/10.1016/S01697439(98)00055-0 


\section{AUTHORS}

Chenglin Sui, is with Beijing University of Chinese Medicine, and the Key Laboratory of TCM-Information Engineering of State Administration of Traditional Chinese Medicine, Beijing, 100102, China (e-mail: chenglinsui@126.com).

Yanfang Peng, is with Beijing University of Chinese Medicine, and the Key Laboratory of TCM-Information Engineering of State Administration of Traditional Chinese Medicine, Beijing, 100102, China (e-mail: 283485436@qq.com).

Luwei Zou, is with Beijing University of Chinese Medicine, and the Key Laboratory of TCM-Information Engineering of State Administration of Traditional Chinese Medicine, Beijing, 100102, China (e-mail: 770939553@qq.com).

Yanling Pei, is with Beijing University of Chinese Medicine, and the Key Laboratory of TCM-Information Engineering of State Administration of Traditional
Chinese Medicine, Beijing, 100102, China (e-mail: 894359125@qq.com).

\section{CORRESPONDING AUTHORS:}

Xinyuan Shi, is with Beijing University of Chinese Medicine, and the Key Laboratory of TCM-Information Engineering of State Administration of Traditional Chinese Medicine, Beijing, 100102, China (e-mail: shixinyuan01@163.com, Tel.: +86-010-84738621; fax: +86-010-84738621).

Yanjiang Qiao, is with Beijing University of Chinese Medicine, and the Key Laboratory of TCM-Information Engineering of State Administration of Traditional Chinese Medicine, Beijing, 100102, China (e-mail: yjqiao@263.net, Tel.: +86-010-84738621; fax: +86-01084738621).

This article is an extended and modified version of a paper presented at the 2012 International conference on Applied Science and Engineering Innovation (ASEI2012), held in Beijing, China, December 2012. Submitted 21 June 2013. Published as re-submitted by the authors 23 July 2013. 